\title{
Paris, Bibliothèque nationale de France, gr. 1672
}

\author{
I. Costantinopoli, ante 1362; II. Costantinopoli, XV secolo.
}

Comp.; ff. A-F, 1-944, 945, A’-G'; il f. 1 appartiene al primo fascicolo: in origine bianco, nel XV secolo fu riempito da <Giorgio Disypatos Galesiota> con il pinax dell'esemplare. La numerazione di alcuni fogli è doppia: ff. 128bis; 484 bis; 873bis; a partire dal f. 854, numerato erroneamente come f. 864, ha inizio un'altra foliotazione. Sono bianchi i ff. 153v, 869v, 902v-903r, 933r, 945rv. Legatura in marocchino rosso con stemmi di Enrico IV impressi sui piatti anteriore e posteriore e arricchiti con gigli angolari; sul dorso in basso vi è un foglio con la segnatura del codice.

Il manoscritto, composto da due unità codicologiche, è stato realizzato a Costantinopoli prima del 1362, anno in cui Manuele Tzicandile completò a Mistrà una sua copia, oggi Oxon. Canon. gr. 93 + Ambr. D 538 inf. Sono prova dell'origine costantinopolitana del codice la presenza dell'Anonimo G (Pérez Martín 1997b, 400-441), di Niceforo Gregora (Bianconi 2003, n. 130) e di Argiro.

\section{I. ff. 1-936 (Costantinopoli, ante 1362)}

Perg.; fasc. $1^{10-1}(9), 2-16^{8}(128 \mathrm{bis}), 17-19^{8}$ (ff. 136-152), [il fascicolo ventesimo, numerato come tale al f. 153r, è (ri)numerato come ventunesimo sul f. 154r], $20[21]^{8+1}(161), 21[22]^{8}(169)-24[25]^{8}(185)$, $25[26]^{8-1}(200), 26[27]^{6}(206) ; 27[28]^{8}(214), 28[29]^{6}(220), 29[30]-53[54]^{8}(412), 54[55]^{6}(418), 55[56]-$ $56[57]^{8}(434), 56[1]^{8}(442), 57[2]^{6}(448), 58[3]-109[59]^{8}$ (in realtà foglio 856 , ma segnato come 865 ), $111[60]^{4}(869), 112[61]^{8-1}(875), 112[62]-114[64]^{8}(899), 115[65]^{8-1}(906), 114[66]-116[68]^{8}(930), 117[69]^{6}$ (936); doppia fascicolazione: resta traccia sul recto del primo foglio e sul verso dell'ultimo foglio di ogni fascicolo; la prima numerazione si arresta al f. 435, il quale è doppiamente numerato $v \zeta^{\circ}$ e $\alpha^{\prime}$. Dal f. 435 ha inizio una seconda numerazione, che termina al f. 936v, segnato come $\xi \theta^{\prime} ; \mathrm{mm}$ 402 x 295 = 53 // 270 // 95 x 33// 86 // 20 // 85 // 10 // 72; due colonne, unità di rigatura 7; rigatura tracciata a secco, sistema S/L 1, tipo S/L 10D2n (Muz 1-2-11/ 0/0/J); 11. 40/rr. 40; 42/rr. 42; resta traccia della foratura.

\section{Scrittura}

Nell'unità si distinguono tre copisti: <Anonimo G (Bianconi 2003, 553; Pérez Martín 2008, 401)> (ff. 2r-706v, 870r-875v); <Isacco Argiro> (ff. 707r-869v); <Manuele Tzicandile (RGK III, nr. 419)> (ff. 876-936v). 


\section{Contenuto}

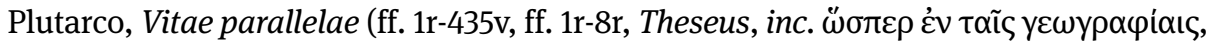

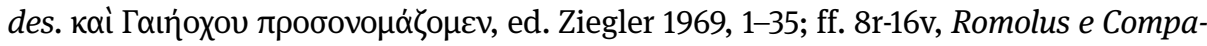

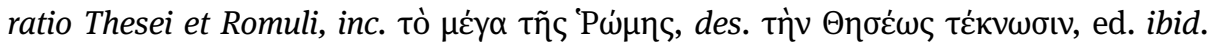

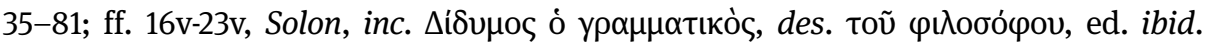

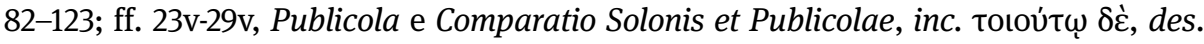

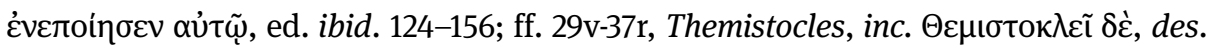

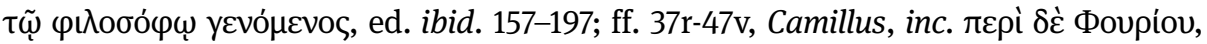

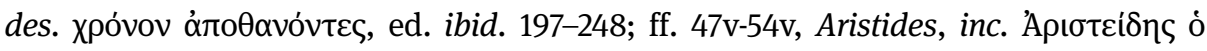

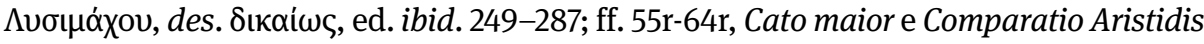

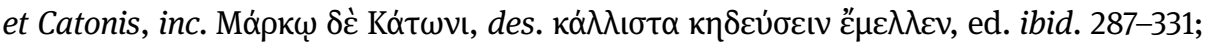

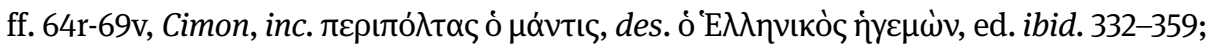

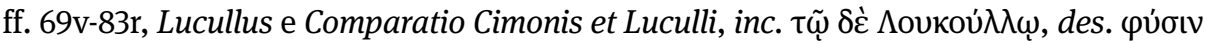

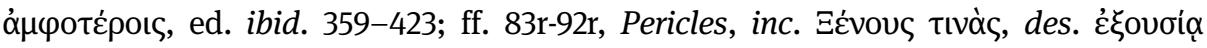

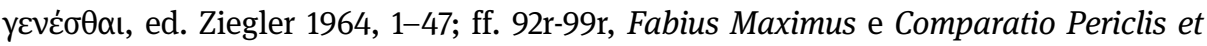

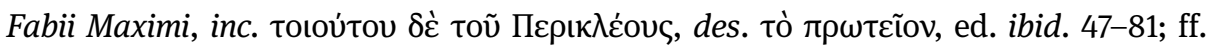

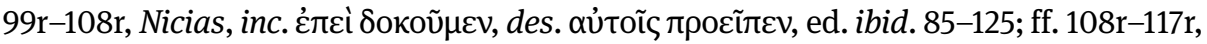

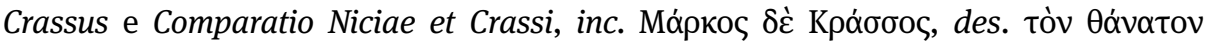

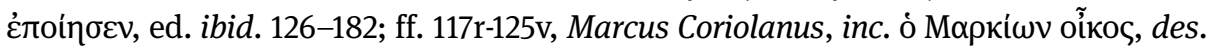

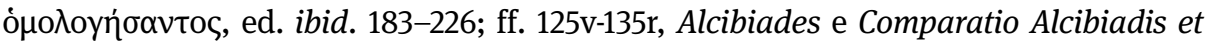

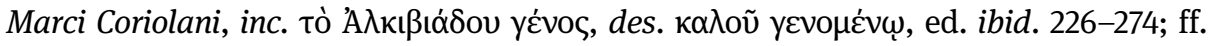

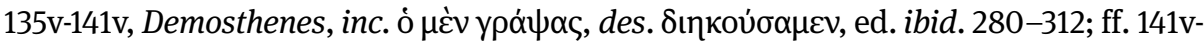

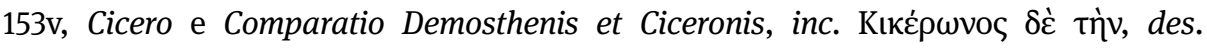

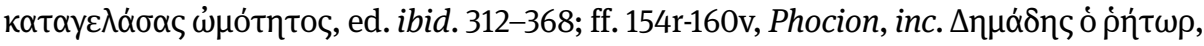

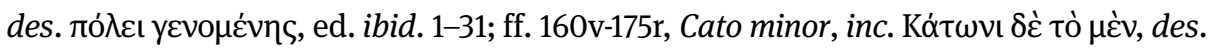

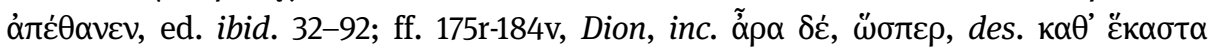

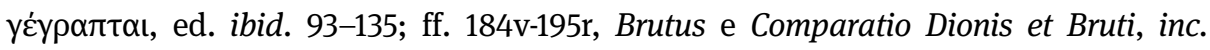

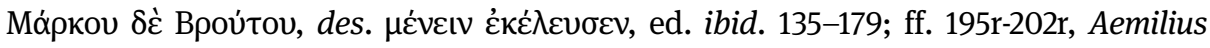

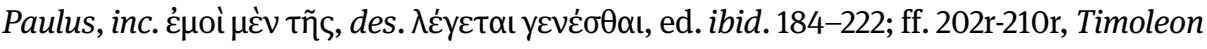

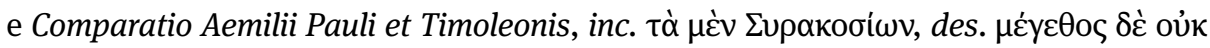

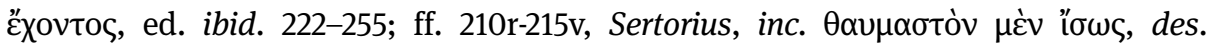

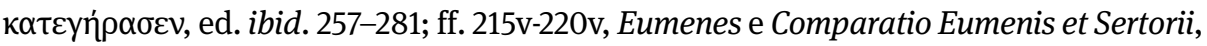

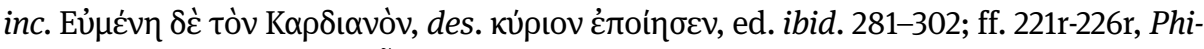

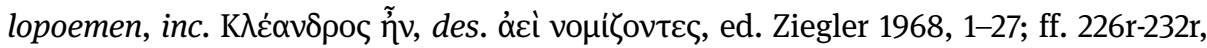
Titus Flaminius e Comparatio Philopoemenis et Titi Flaminis, inc. ōv $\pi \alpha \rho \alpha \beta \alpha \dot{\lambda} \lambda \mathrm{\alpha} \mu \varepsilon v$, des.

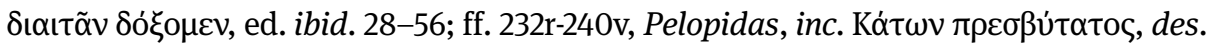

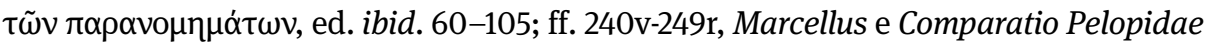

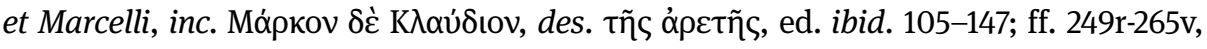

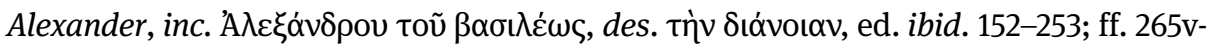

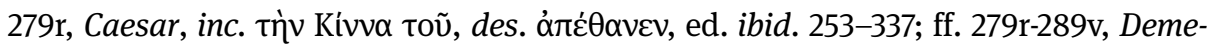

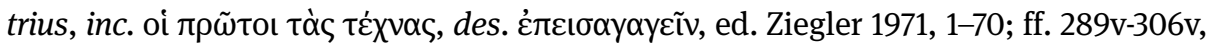




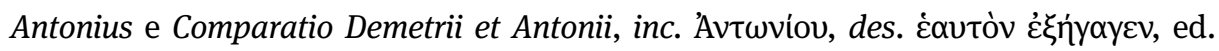

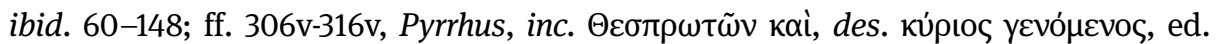

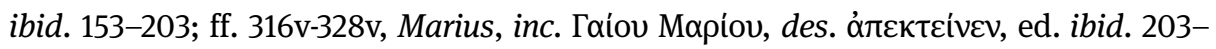

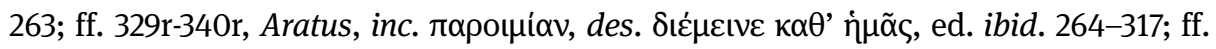

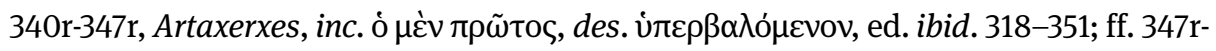

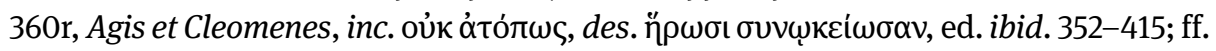
360r-369v, Tiberius et Caius Graccus e Comparatio Agidis et Cleomenis cum Tiberio et

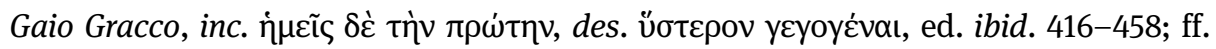

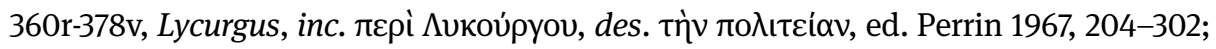

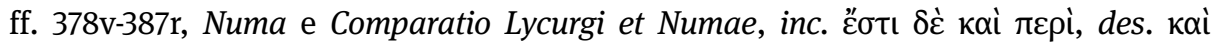

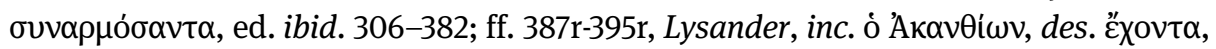
ed. Perrin 1968, 234-320; ff. 395r-406v, Sulla e Comparatio Lysandri et Sullae, inc.

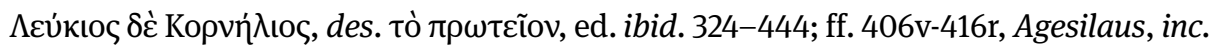

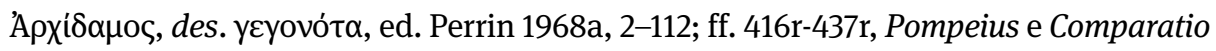

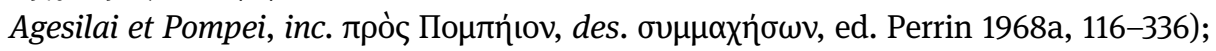

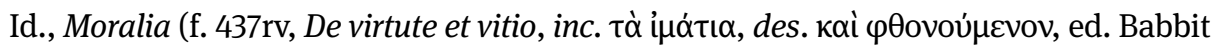

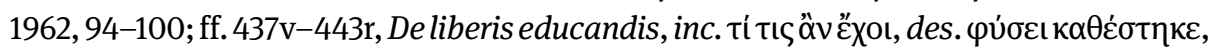
ed. ibid. 4-68; ff. 443r-448r, Quomodo quis suo in virtute sententiat profectus, inc. Tís

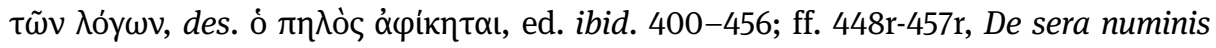

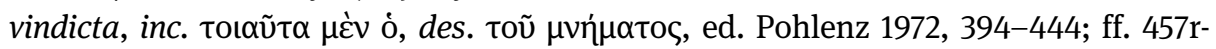

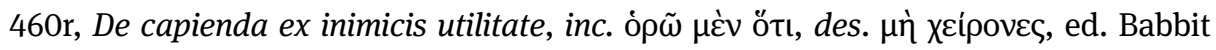
1962, 4-40; ff. 460r-469r, Quomodo adolescens poetas audire debeat, inc. cỉ $\mu \varepsilon \dot{v}$, ஸ்

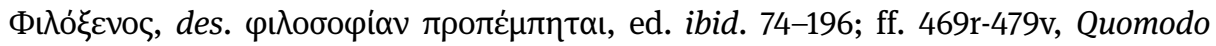

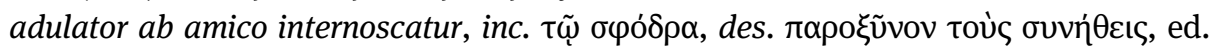

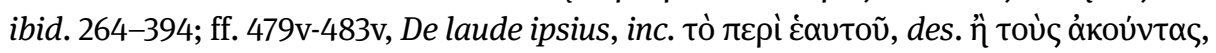

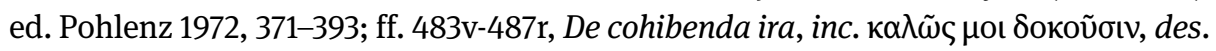

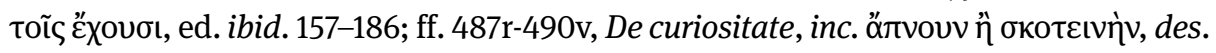

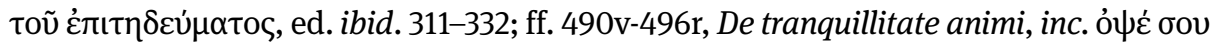

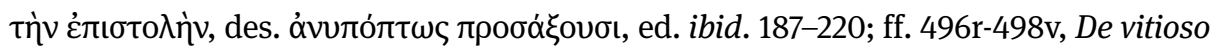

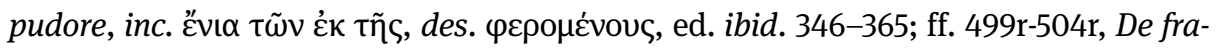

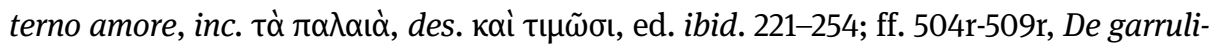

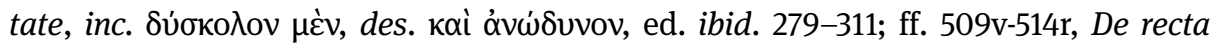

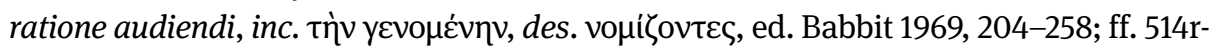

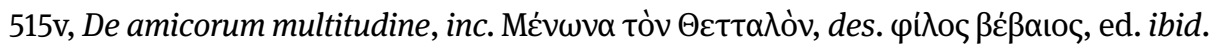

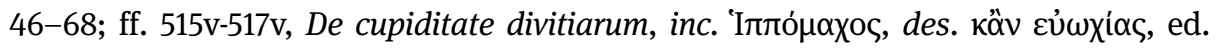

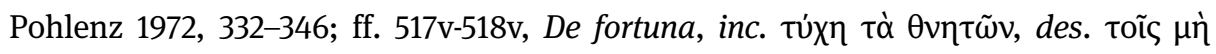

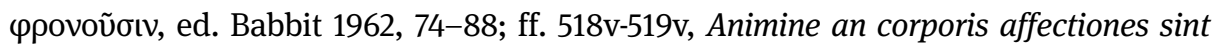

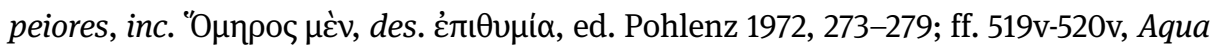

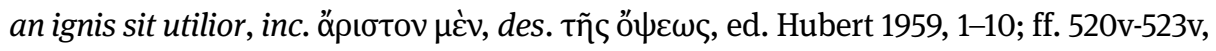

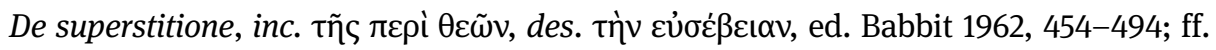

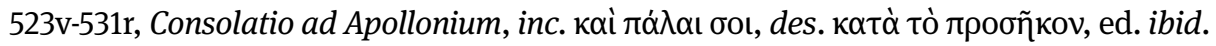

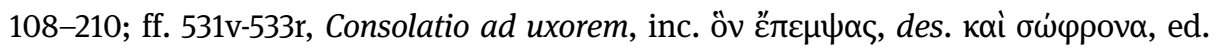




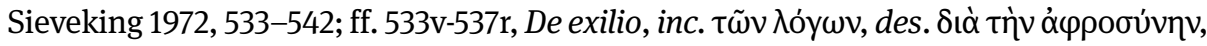

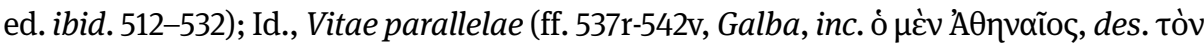

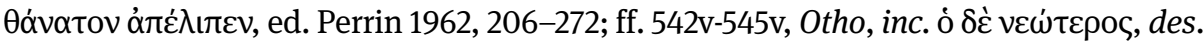

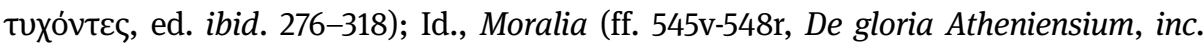

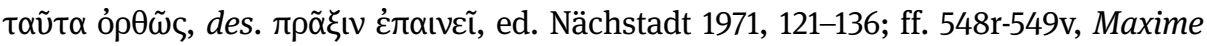

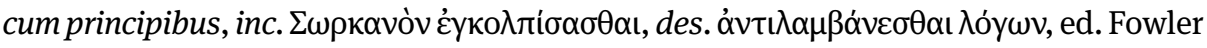

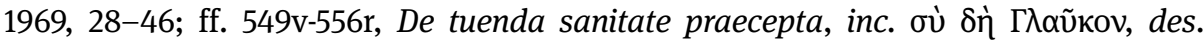

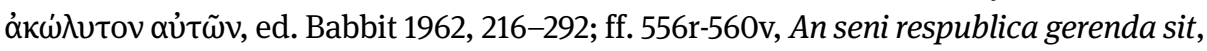

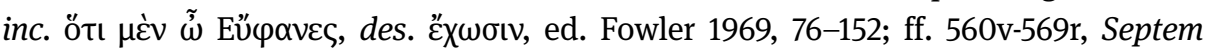

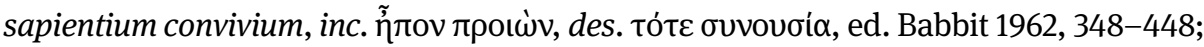

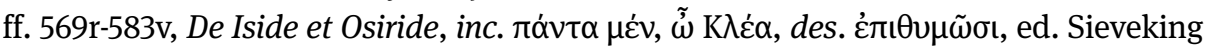

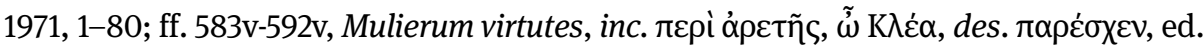

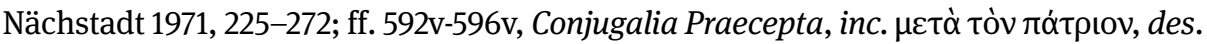

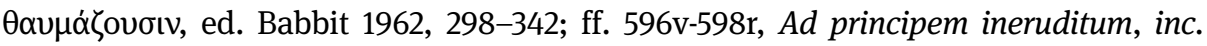

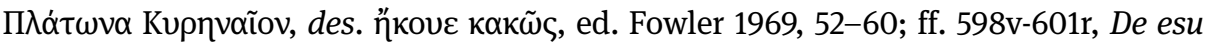

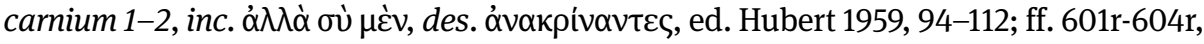

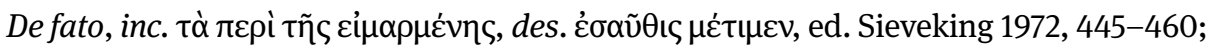

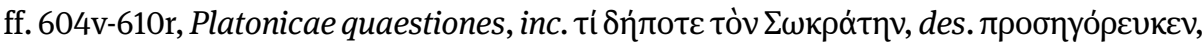

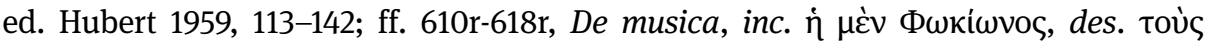

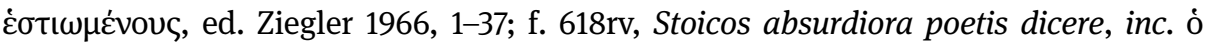

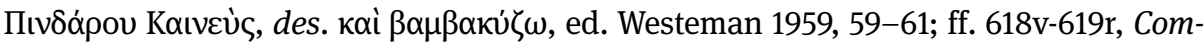

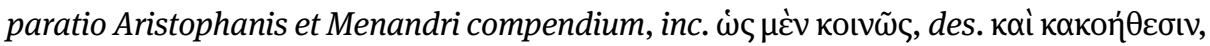

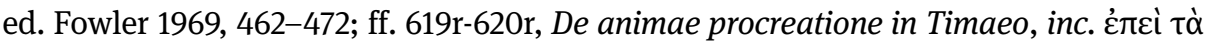

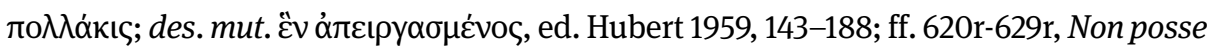

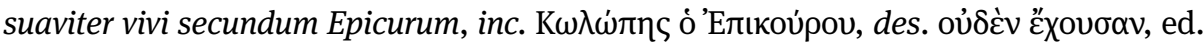

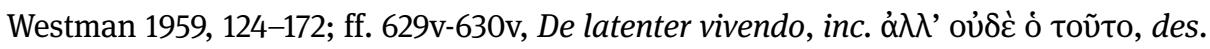

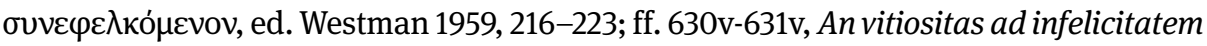

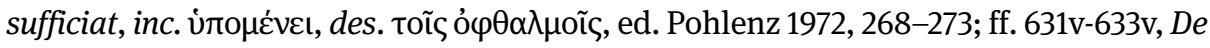

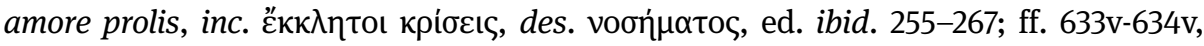

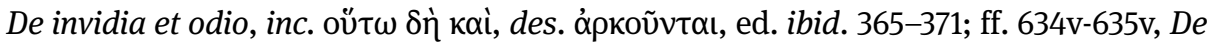

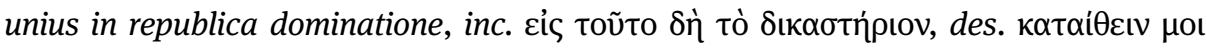

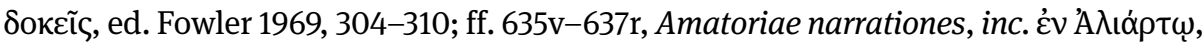

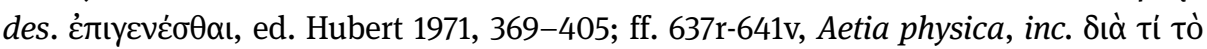

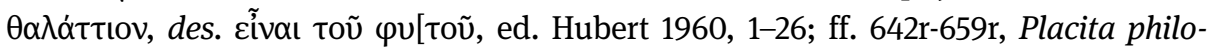

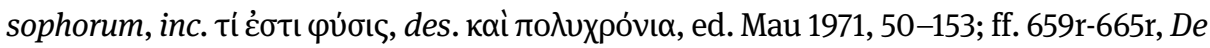

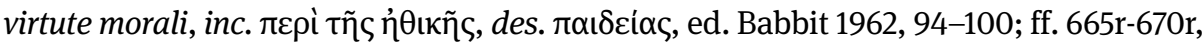

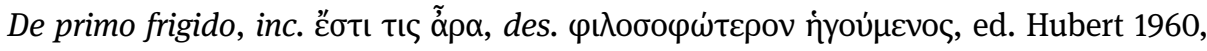

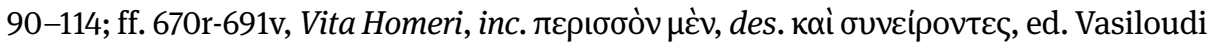

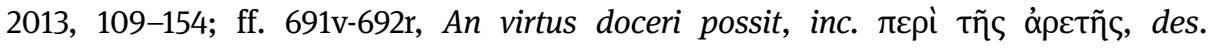

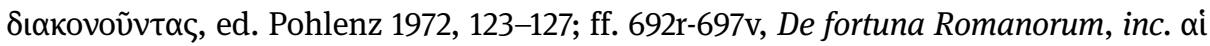

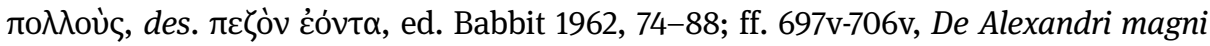




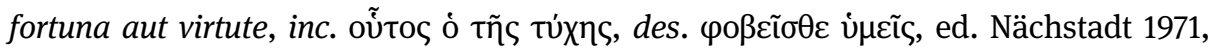

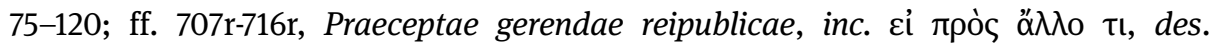

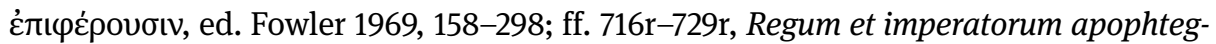

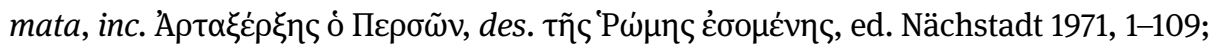

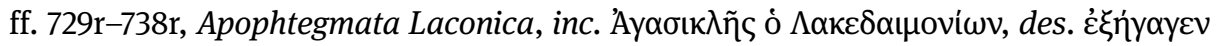

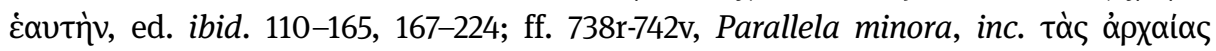

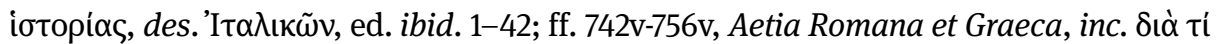

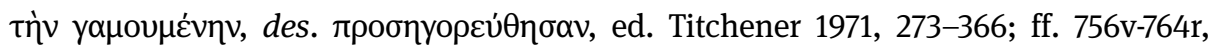

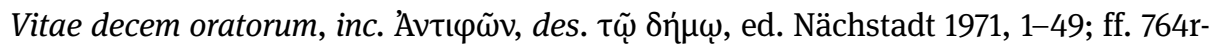

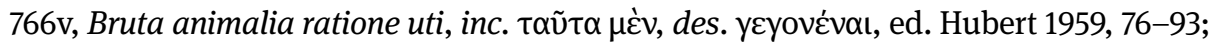

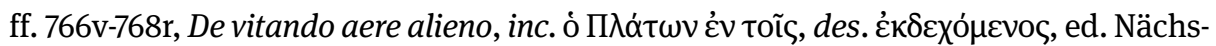

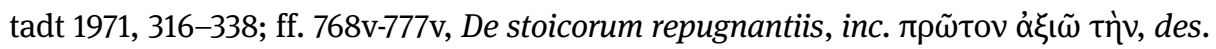

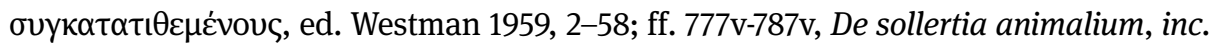

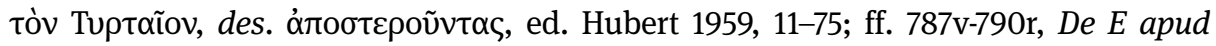

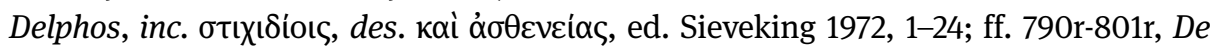

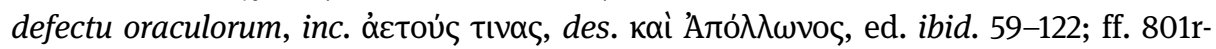

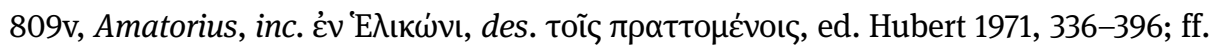

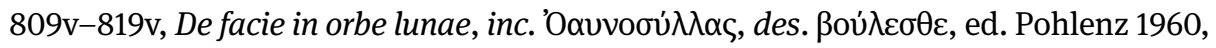

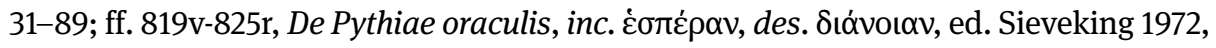

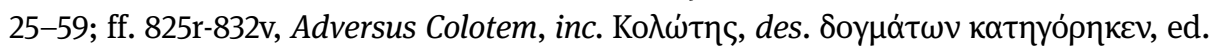
Westman 1959, 173-215; ff. 832v-843r, De communibus notitiis contra Stoicos, inc. ooì $\mu \varepsilon \dot{v}$

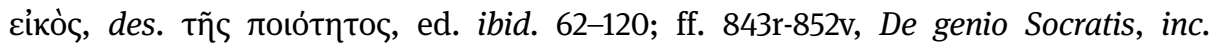

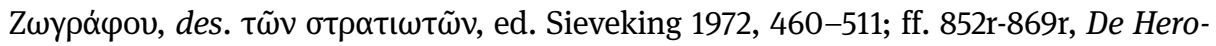

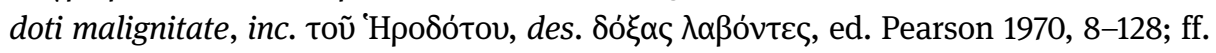

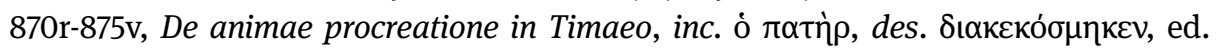

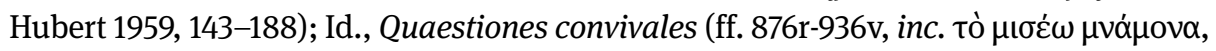

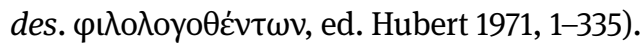

\section{II. ff. 1*; 937r-944v (Costantinopoli, XV secolo)}

Perg.; fasc. $1^{8}$ (937-944); num. fasc. assente; mm 402 x $295=53$ // 270 // 95 x 33// 86 // 20 // 85 // 10 // 72; due colonne, unità di rigatura 7; rigatura tracciata a secco, sistema S/L 1, tipo S/L 10D2n (Muz 1-2-11/0/0/J); 11. 40/rr. 40; 42/rr. 42.

\section{Scrittura}

In questi fogli è presente un solo copista: A(ff. 1*; 937r-944v) identificato con <Giorgio Disypatos Galesiota> (RGK III nr. 99). 


\section{Contenuto}

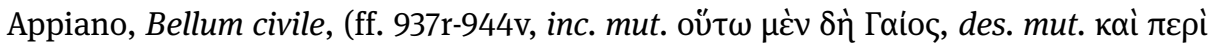

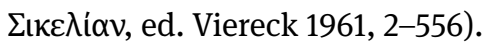

\section{Storia del codice}

Dopo essere stato copiato a Costantinopoli, il codice fu probabilmente portato da Manuele Tzicandile a Mistrà, dove ne realizzò la copia Oxon. Canon. gr. 93 + Ambr. D 538 inf.. In seguito, il codice è appartenuto a Giorgio Disypato Galesiota, che vi aggiunse, oltre al pinax iniziale, alcuni excerpta dall'Historia romana di Appiano. Dalla Biblioteca del Serraglio nel 1687 fu acquistato dall'ambasciatore Pierre de Girardin per il re di Francia (cf. Martinelli Tempesta 2006a, 75-76).

\section{Bibliografia}

\section{Cataloghi}

Caballero (1989) 41-42; Hahn (1905) 43-128; Omont (1888) 120-121.

\section{Edizioni}

Babbit (1962); Babbit (1969); Fowler (1969); Hubert (1959); Hubert (1960); Hubert (1971); Mau (1971); Nächstadt (1971); Pearson (1970); Perrin (1962); Perrin (1967); Perrin (1968); Perrin (1968a); Pohlenz (1960); Pohlenz (1972); Sieveking (1972); Titchener (1971); Vasiloudi (2013); Viereck (1961); Westman (1959); Ziegler (1964); Ziegler (1966); Ziegler (1968); Ziegler (1969); Ziegler (1971).

\section{Codicologia e paleografia}

Bianconi (2003) 552-554; Manfredini (1996) passim; Manfredini (1989) 130-131; Manfredini (2000) 661-663; Smith (1975) 49; Pérez Martín (1996) 400-402; Martinelli Tempesta (2006a) 72-76; Vendruscolo (1994) 32-33, 38-41, 83-85; Wilson (1975) 76.

\section{Riproduzioni}

Hansen (1969) tavv. 1-2. 